\title{
Análise dos compostos bioativos e características físico-químicas de berinjela e tomate cereja em produção agroecológica
}

\author{
Shênia Santos Monteiro ${ }^{1}$, Shirley Santos Monteiro ${ }^{2}$, Elnatan Alves da Silva ${ }^{2}$ \\ ${ }^{1}$ Universidade Federal de Campina Grande; shenia-monteiro@ @otmail.com; ${ }^{2}$ Universidade Federal da Paraíba; \\ shirley_pinto_monteiro@hotmail.com; alveselnatan@gmail.com.
}

\begin{abstract}
RESUMO: A berinjela e o tomate são os frutos mais produzidos e consumidos no Brasil, em decorrência das características sensoriais e nutricionais. A crescente demanda por alimentos agroecológicos e a carência de informações a respeito do impacto que o sistema de produção gera na qualidade pós-colheita do produto final e consequentemente na escolha por parte do consumidor. Diante disso, o presente estudo teve como objetivo caracterizar a berinjela e o tomate cereja agroecológico quanto aos parâmetros físico-químicos e compostos bioativos. $\mathrm{O}$ experimento foi realizado no Setor de Agricultura da Universidade Federal da Paraíba, Campus-III, Bananeiras-PB, utilizando um delineamento inteiramente casualizado, com quatro repetições. Foram avaliadas as variáveis: cor, potencial hidrogeniônico (pH), acidez total titulável (\% de ác. cítrico), sólidos solúveis totais ( ${ }^{\circ}$ Brix), ratio SS/AT (\%), ácido ascórbico (mg.100.g ${ }^{-1}$ ), compostos fenólicos totais (mg.EAG.100.g ${ }^{-1}$ ) e atividade antioxidante (g/g de DPPH). Os resultados obtidos ao analisar os frutos de berinjela e tomate cereja mostraram boa qualidade nutricional de acordo com as peculiaridades de cada fruto. Com isto, pode-se concluir que os frutos avaliados apresentaram propriedades nutricionais, podendo ser considerados como alimentos funcionais, decorrente da presença de compostos bioativos, revelando efeito positivo do sistema de produção agroecológico sobre a qualidade dos frutos.
\end{abstract}

PALAVRAS-CHAVE: Atividade antioxidante; Compostos fenólicos; Solanum melongena L; Solanum lycopersicum L. var. cerasiforme.

\section{INTRODUÇÃO}

A berinjela e o tomate cereja pertence à família das solanáceas, a mesma família de outras hortaliças de grande importância socioeconômica, dentre as quais se destacam a batata (Solanum tuberosum L.) e o pimentão (Capsicum annuит Group L.) (REIS, 2006)

A berinjela e o tomate cereja é consumida em todo o mundo, principalmente em países tropicais e subtropicais, com uma produção mundial de cerca de 49 milhões de toneladas (FAO, 2016). A procura de produtos mais saudáveis pelos consumidores se deve as suas propriedades nutricionais. Consequentemente, a demanda por produtos oriundos de manejo agroecológico e orgânico tem-se difundido nos últimos anos, uma vez que, esses alimentos têm mostrado qualidade nutricional superior a alimentos produzidos em outros sistemas de produção (TENUTA, 2017; SAATH; FACHINELLO, 2018). Além disso, a produção de alimentos sob sistemas agroecológicos contribui para minimização dos danos ao meio ambiente, causado pelo uso inadequado de agrotóxicos.

A berinjela é rica em vitaminas do complexo B: ácido pantotênico (vitamina B5), piridoxina (vitamina B6), tiamina (vitamina B1) e niacina. É também conhecida por apresentar propriedades medicinais, sendo indicada para pacientes diabéticos e no auxílio no tratamento de doenças no fígado (SHUKLA; NAIK, 1993).

O tomate cereja possui tamanho pequeno têm sabor delicado e suculento, e são excelentes para a alimentação fresca, que geralmente é feita em salada (LIU et al., 2018). O cultivo do tomate cereja tem aumentando, em decorrência, principalmente as características sensoriais, ocasionando aumento da procura e aceitabilidade dos frutos pelo mercado consumidor. Os tomates cultivados agroecológicos apresentam maior teor de ácidos ascórbico e fenólicos totais do que os tomates convencionais, além de maior atividade antioxidante (BORGUINI et al., 2013). No entanto, os compostos antioxidantes tornam-se mais eficazes quando consumidos in natura do que quando ingerido isoladamente, provavelmente devido ao efeito sinérgico com outros compostos presentes neste fruto (BOHN et al., 2013).

Assim, considerando a crescente demanda por alimentos agroecológicos e a carência de informações a respeito do impacto que o sistema de produção gera na qualidade do produto final, objetivou-se com este estudo caracterizar a berinjela e o tomate cereja agroecológico quanto a qualidade físico-química e compostos bioativos.

\section{MATERIAL E MÉTODOS}

As berinjelas e os tomates cereja foram produzidos em cultivo agroecológico na área experimental do Setor de Agricultura do Centro de Ciências Humanas, Sociais e Agrárias, pertencente a Universidade Federal da Paraíba, Campus - III, Bananeiras - PB.

Os frutos foram coletados em estádios de maturação comercial e transportados ao Laboratório de Fisiologia PósColheita, onde efetuou-se a seleção para obtenção de lotes uniformes, eliminando-se os frutos com defeitos. 
MONTEIRO, S. S. et al. Análise dos compostos bioativos e características físico-químicas de berinjela e tomate cereja em produção agroecológica. In: II Congresso Paraibano de Agroecologia \& IV Exposição Tecnológica, 2019. Anais... Caderno Verde de Agroecologia e Desenvolvimento Sustentável, Pombal, v. 9, n.7, e-6927, 2019.

Posteriormente, foram lavados em água potável, para retirada das sujidades oriundas do campo, seguida da imersão em solução de hipoclorito de sódio a 200 ppm por 10 minutos e secos à temperatura ambiente.

As avaliações foram realizadas quanto aos parâmetros físico-químicos: cor, realizada através de Colorímetro Delta Color (Delta Vista d.0) do tipo refletância, o qual foi previamente calibrado, com fonte de iluminação de D65 e ângulo de observação de $10^{\circ}$; químicas: $\mathrm{pH}$ foi determinado utilizando-se um potenciômetro digital de bancada, teor de sólidos solúveis totais foi mensurado em um refratômetro digital, a qual o resultado foi expressos em ${ }^{\circ} \mathrm{Brix}$, acidez total titulável, medida em $10 \mathrm{~g}$ de polpa, homogeneizado em $50 \mathrm{~mL}$ de água destilada e posteriormente titulada com $\mathrm{NaOH}$ 0,1N. A relação em acidez total titulável e sólidos solúveis totais, cujo resultados foi expresso em percentual; compostos bioativos: ácido ascórbico $\left(\mathrm{mg} .100 . \mathrm{g}^{-1}\right)$, determinado através de espectrofotômetro (COX; PEARSON, 1976), que consiste na reação do ácido ascórbico com o 2,6-diclorofenol indofenol. A leitura realizada em espectrofotômetro a $520 \mathrm{~nm}$ e os resultados expressos em $\mathrm{mg}$ de ácido ascórbico por $100 \mathrm{~g}$ de berinjela e tomate cereja. Os polifenóis extraíveis totais foram definidos conforme descrito por Waterhouse (2002). Tomou-se 0,2 g de material vegetal, adicionando $4 \mathrm{~mL}$ de metanol $50 \%$ e deixando para extrair por 1 hora. Em seguida, a mistura foi centrifugada a $15.000 \mathrm{rpm}$ durante $30 \mathrm{~min}$. Posteriormente, as amostras foram mensuradas em espectrofotômetro através da absorbância a $765 \mathrm{~nm}$. Os resultados foram expressos em equivalente de ácido gálico (mg.EAG.100.g ${ }^{-1}$ de extrato de berinjela e tomate cereja). A atividade antioxidante total foi determinada através do método DPPH, utilizando-se o reativo 2,2-diphenil-1-picrilhidrazil (BRAND-WILLIAMS et al., 1995). A partir do extrato obtido para os polifenóis extraíveis totais, foram preparadas três diluições diferentes $\left(100,50\right.$ e $\left.10 \mu \mathrm{g} \cdot \mathrm{mL}^{-1}\right)$ de acordo com a metodologia de Rufino et al., (2007).

O delineamento utilizado foi o inteiramente casualizado, com quatro repetições e cinco frutos por parcela. Os resultados obtidos foram submetidos ao teste Tukey através do programa estatístico SAS® (2013) licenciado pela Universidade Federal da Paraíba.

\section{RESULTADOS E DISCUSSÃO}

Na Tabela 1, encontra-se os resultados obtidos quanto aos parâmetros de cor da berinjela e tomate cereja em estádio comercial.

Tabela 1. Parâmetros de cor seguindo dos seus respectivos desvios padrões em berinjela e tomate cereja agroecológica

\begin{tabular}{ccc} 
& Berinjela & Tomate Cereja \\
\hline $\mathrm{L}^{*}$ & $19,13 \pm 3,10 \mathrm{~b}$ & $39,80 \pm 2,25 \mathrm{~b}$ \\
$\mathrm{a}^{*}$ & $0,34 \pm 1,12 \mathrm{~b}$ & $17,61 \pm 2,84 \mathrm{~b}$ \\
$\mathrm{~b}^{*}$ & $-2,27 \pm 1,23 \mathrm{~b}$ & $19,4 \pm 4,02 \mathrm{~b}$ \\
$\mathrm{C}$ & $2,70 \pm 0,85 \mathrm{~b}$ & $26,03 \pm 2,40 \mathrm{~b}$ \\
$\mathrm{~h}^{\circ}$ & $271,17 \pm 4,18 \mathrm{a}$ & $52,15 \pm 4,25 \mathrm{a}$ \\
\hline
\end{tabular}

*Média \pm desvio padrão seguidas por letras iguais na coluna não diferem entre si pelo teste de Tukey a 5\% de probabilidade.

O índice $\mathrm{a}^{*}$ pode ir de vermelho (valor positivo) ao verde (valor negativo), os valores encontrados demostrando a coloração vermelha do tomate cereja, a qual se dever a pigmentos, com os carotenoides, em particular o licopeno, conferindo a cor vermelha ao fruto (JAVANMARDI; KUBOTA, 2006). Já as berinjelas apresentaram valor positivo, o que se deve as antocianinas, a principal antocianina encontrada na casca da berinjela é nasunim, foi isolada pela primeira vez por Kuroda e Wada (1933).

A cor do fruto é um parâmetro importante na classificação do produto industrializado, sendo necessário que o fruto apresente coloração roxa-intensa e uniforme da parte externa do fruto (CLEMENTE; BOITEUX, 2012).

A respeito do teor de $b^{*}$ podem ir de amarelo (valor positivo) ao azul (valor negativo), os tomates cereja apresentaram tons de amarelos mais acentuados, o qual se deve a degradação da clorofila para formação de outros pigmentos. Já a berinjela apresentou diminuição do valor de $b^{*}$, podendo esse comportamento ser em decorrência da variação entre frutos e diminuição do teor de água (OLIVEIRA et al., 2016b).

Acerca da cromaticidade (C), apresentou variação nos valores obtidos. Os tomates cereja se apresentavam, indicando que os frutos tenderam a apresentar intensidade da cor predominante, mostraram diferença entre a berinjela (Tabela 1). Para a tonalidade dos frutos, representada pelo $\mathrm{h}^{\circ}$, as berinjelas apresentaram os maiores valores, ao relacionar com a tomate cereja com menores valores.

Os frutos do tomate cereja avaliados por Sousa et al. (2015) apresentaram coloração tendendo levemente ao amarelo e fortemente ao vermelho e com alta luminosidade. Os resultados obtidos no presente estudo se assemelha aos obtidos quando avaliou-se o tomate cereja cultivado no sistema agroecológico.

Na Tabela 2, encontra-se os valores obtidos quanto as características físico-químicas e presença de compostos bioativos na berinjela e tomate cereja em estádio comercial. 
MONTEIRO, S. S. et al. Análise dos compostos bioativos e características físico-químicas de berinjela e tomate cereja em produção agroecológica. In: II Congresso Paraibano de Agroecologia \& IV Exposição Tecnológica, 2019. Anais... Caderno Verde de Agroecologia e Desenvolvimento Sustentável, Pombal, v. 9, n.7, e-6927, 2019.

Tabela 2. Valores de potencial hidrogeniônico, acidez total titulável, sólidos solúveis totais, ratio SS/AT, ácido ascórbico, compostos fenólicos totais e atividade antioxidante seguindo dos seus respectivos desvios padrões.

\begin{tabular}{lcc}
\hline & Berinjela & Tomate Cereja \\
\hline Potencial Hidrogeniônico $(\mathrm{pH})$ & $4,41 \pm 0,09 \mathrm{a}$ & $3,86 \pm 0,07 \mathrm{~b}$ \\
Acidez Total Titulável $(\%$ de ác. cítrico) & $0,145 \pm 0,01 \mathrm{~b}$ & $0,308 \pm 0,05 \mathrm{a}$ \\
Sólidos Solúveis Totais ( ${ }^{\circ}$ Brix) & $4,09 \pm 0,23 \mathrm{a}$ & $4,88 \pm 0,42 \mathrm{~b}$ \\
Ratio Acidez Total Titulável/Sólidos Solúveis Totais $(\%)$ & $28,26 \pm 1,08 \mathrm{a}$ & $16,16 \pm 2,35 \mathrm{~b}$ \\
Ácido Ascórbico $\left(\mathrm{mg} .100 . \mathrm{g}^{-1}\right)$ & $9,74 \pm 0,08 \mathrm{~b}$ & $9,85 \pm 0,05 \mathrm{a}$ \\
Compostos Fenólicos Totais (mg.EAG.100.g $\left.{ }^{-1}\right)$ & $14,66 \pm 1,84 \mathrm{~b}$ & $26,76 \pm 0,90 \mathrm{a}$ \\
Atividade Antioxidante (g/g de DPPH) & $509,58 \pm 2,12 \mathrm{a}$ & $456,85 \pm 1,70 \mathrm{a}$ \\
\hline
\end{tabular}

*Média \pm desvio padrão seguidas por letras iguais na coluna não diferem entre si pelo teste de Tukey a $5 \%$ de probabilidade.

Os valores médios do pH, para ambos os frutos estudados, estão em concordância com valores considerados ideais para o produto analisado, caracterizando-os como frutos ácidos, sendo o tomate cereja mais ácido que a berinjela. A característica ácida ajuda na conservação do fruto após a colheita (NASCIMENTO et al., 2013).

Para a acidez total titulável, apresentaram efeito significativo $(\mathrm{p}<0,05)$ nas frutas avaliadas, os maiores teores de acidez total titulável foi constatado no tomate cereja. Chiumarelli e Ferreira (2006) avaliando a qualidade pós-colheita de tomate encontrou valores de acidez total titulável de $0,33 \%$, valores semelhantes aos encontrados neste estudo. Tal comportamento pode ter sido influenciado pela resposta do metabolismo ao ambiente e sistema de cultivo (OLIVEIRA et al., 2016a).

Os teores de sólidos solúveis totais divergiram estatisticamente $(p<0,05)$, o maior teor foi identificado na berinjela de 4,09 ${ }^{\circ}$ Brix, o qual influenciam de forma positiva no sabor das hortaliças. Resultado semelhante foi observado por Kaynas et al. (1995), em que o aumento no teor de sólidos solúveis totais em berinjelas pode ter ocorrido devido ao aumento do metabolismo do amido que é hidrolisado em compostos de menor peso molecular como açúcares solúveis (PINSETTA JUNIOR, 2018).

No que se refere ao ratio de SST/ATT, a berinjela apresentou maior ratio SST/ATT em relação ao tomate cereja. O ratio é uma das melhores formas de avaliação do sabor, maturação e palatabilidade dos frutos, o qual ocorre, em grande parte, devido ao balanço de ácidos orgânicos e açúcares, sendo mais representativo que a mensuração destes parâmetros isoladamente. Quando esses valores são altos, significa que o fruto está em bom grau de maturação, pois o mesmo aumenta quando há decréscimo de acidez e alto conteúdo de SST, decorrentes da maturidade (PEREIRA et al., 2006).

Da Tabela 2, o teor de ácido ascórbico da berinjela e tomate cereja, observou-se que o valor encontrado diferiu significativamente $(\mathrm{p}>0,5)$, indicando a modificação de acordo com os frutos estudados. No entanto, o tomate cereja apresentou maiores teores de ácido ascórbico em relação a berinjela.

Del Aquila et al. (2006) citam que o teor de ácido ascórbico nos produtos frescos, é afetado tanto pelas reações de biossíntese como de degradação do ácido ascórbico. Ferreira et al. (2010) citam que o teor de vitamina C durante o amadurecimento de tomate é reflexo da translocação contínua e síntese do ácido ascórbico proveniente do acúmulo de sólidos solúveis totais e açúcares redutores entre os estádios de maturação.

Os teores de polifenóis extraíveis totais apresentou maior valor nos frutos de tomates cereja em relação aos frutos de berinjelas. Os valores podem variar de acordo com os fatores abióticos como luz, temperatura e condições de cultivo podem induzir um aumento no conteúdo de polifenóis nos vegetais (GONZALEZ-AGUILAR et al., 2012).

A síntese de compostos fenólicos pode ser vista de maneira positiva em termos nutricionais. Há muitos benefícios para a saúde com o consumo de alimentos ricos em polifenóis, podendo estes atuar como antioxidantes removendo radicais livres ou quebrando cadeias, dependendo de suas estruturas químicas (MCSWEENEY; SEETHARAMAN, 2015), dando sabor e cor aos alimentos.

No que se refere à atividade antioxidante, não divergiram estatisticamente entre os frutos. Os antioxidantes dos frutos são substâncias que podem prevenir ou reparar os danos oxidativos causados por espécies reativas de oxigênio em lipídios, proteínas e ácidos nucleicos (VASCONCELOS et al., 2014).

A capacidade de atender ao desejo do consumidor contemporâneo, que anseia ter acesso a todos os benefícios que os alimentos agroecológicos podem proporcionar na qualidade de vida, decorrentes de vários efeitos metabólicos e fisiológicos que contribuem para um melhor desempenho do organismo que os consome (AZEVEDO; PELICIONI, 2011; CARVALHO; LINO, 2014).

\section{CONCLUSÕES}

Os resultados obtidos diante o estudo dos frutos de berinjela e tomate cereja em produção agroecológica, mostraram boa qualidade nutricional dos frutos, podendo serem considerados como alimentos funcionais, decorrente da presença de compostos bioativos, revelando efeito positivo do sistema de produção agroecológico sobre a qualidade dos frutos. 
MONTEIRO, S. S. et al. Análise dos compostos bioativos e características físico-químicas de berinjela e tomate cereja em produção agroecológica. In: II Congresso Paraibano de Agroecologia \& IV Exposição Tecnológica, 2019. Anais... Caderno Verde de Agroecologia e Desenvolvimento Sustentável, Pombal, v. 9, n.7, e-6927, 2019.

\section{REFERÊNCIAS}

AZEVEDO, E.; PELICIONI, M. C. F. Promoção da saúde, sustentabilidade e agroecologia: uma discussão intersetorial. Saúde Sociedade, São Paulo-SP, v.20, n.3, p.715-729, 2011.

BOHN, T. BLACKWOOD, M.; FRANCIS, D.; TIAN, Q.; SCHWARTZ, S. J.; CLINTON, S. K. Bioavailability of phytochemical constituents from a novel soy fortified lycopene rich tomato juice developed for targeted cancer prevention trials. Nutrition and cancer, Colorado-EUA, v.65, n.6, p.1-20, 2013.

BORGUINI, R. G.; BASTOS, D. H. M.; MOITA-NETO, J. M.; CAPASSO, F. S.; TORRES, E. A. F. D. Antioxidant potential of tomatoes cultivated in organic and conventional systems. Brazilian Archives of Biology and Technology, Curitiba-PR, v.56, n.4, p.521-529, 2013.

BRAND-WILLIAMS, W.; CUVELIER, M. E.; BERSET, C. Use of a free radical method to evaluate antioxidant activity. Lebensmittel-Wissenschaft and Technologie, Georgia- EUA, v.28, p.25-30, 1995.

CARVALHO, M. M. S.; LINO, L. L. A. Avaliação dos fatores que caracterizam a berinjela (Solanum melongena L.) com um alimento funcional. Nutrire: Revista da Sociedade Brasileira de Alimentação e Nutrição, São Paulo-SP, v.39, n.1, p.130-143, 2014.

CHIUMARELLI, M.; FERREIRA, M. D. Qualidade pós-colheita de tomates 'Débora' com utilização de diferentes coberturas comestíveis e temperaturas de armazenamento. Horticultura Brasileira, Brasília-DF, v.24, n.3, p.381-385, 2006.

CLEMENTE, F. M. V. T.; BOITEUX, L. S. Produção de tomate para processamento industrial. Brasília: EMBRAPA, Brasília-DF, 344 p., 2012.

COX, H. E.; PEARSON, D. Técnicas de laboratório para el analisis de alimentos. Zaragoza: Acríbia, 331 p., 1976.

DEL AQUILA, J. S.; SASAKI, F. F.; HEIFFIG, L. S.; ORTEGA, E. M. M.; JACOMINO, A. P.; KLUGR, R. A. Freshcut radish using different cut types and storage temperatures. Postharvest Biology and Technology, v.40, p.149-154, 2006.

FAO - Food and Agriculture Organization of the United Nations. Disponível em: http://faostat.fao.org. Acesso em: $10 / 07 / 2016$

FERREIRA, S. M. R.; QUADROS, D. A.; KARKLE, E. N. L.; LIMA, J. J.; TULLIO, L. T.; FREITAS, R. J. S. Qualidade pós-colheita do tomate de mesa convencional e orgânico. Ciência e Tecnologia de Alimentos, v. 30, n. 4, p. $858-864,2010$.

GONZALEZ-AGUILAR, G. A.; VILLA-RODRIGUEZ, J. A.; AYALA-ZAVALA, J. F.; YAHIA, E. M. Improvement of the antioxidant status of tropical fruits as a secondary response to some postharvest treatments. Trends in Food Science and Technology, v.21, n.10, p.475-482, 2012.

JAVANMARDI, J.; KUBOTA, C. Variation of lycopene, antioxidant activity, total soluble solids and weight loss of tomato during postharvest storage. Postharvest Biology and Technology, v.41, p.151-155, 2006.

KAYNAS, K.; ÖZELKÖK, S.; SÜMELI, N.; ABAK, K. Controlled and modified atmosphere storage of eggplant (Solanum Melongena L.) fruits. Acta Horticulturae, Brasília-DF, v.412, p.143-151, 1995.

KURODA, C.; WADA, M. The colouring matter of eggplant (Nasunin). Proceedings of the Imperial Academy, Tokyo, n.2, p. 51-52, 1933.

LIU, H. MENG, F.; MIAO, H.; CHEN, S.; YIN, T.; HU, S.; SHAO, Z.; LIU, Y.; GAO, L.; ZHU, C.; ZHANG, B.; WANG, Q. Effects of postharvest methyl jasmonate treatment on main health-promoting components and volatile organic compounds in cherry tomato fruits. Food chemistry, v.263, p.194-200, 2018.

MCSWEENEY, M.; SEETHARAMAN, K. State of polyphenols in the drying process of fruits and vegetables. Critical Reviews in Food Science and Nutrition, Madrid-Spain, v.55, p.660-669, 2015. 
MONTEIRO, S. S. et al. Análise dos compostos bioativos e características físico-químicas de berinjela e tomate cereja em produção agroecológica. In: II Congresso Paraibano de Agroecologia \& IV Exposição Tecnológica, 2019. Anais... Caderno Verde de Agroecologia e Desenvolvimento Sustentável, Pombal, v. 9, n.7, e-6927, 2019.

NASCIMENTO, A. R.; SOARES JÚNIOR, M. S.; CALIARI, M.; FERNANDES, P. M.; RODRIGUES, J. P. M.; CARVALHO, W. T. Qualidade de tomates de mesa cultivados em sistema orgânico e convencional no estado de Goiás. Horticultura Brasileira, Brasília-DF, v.31, n.4, p.628-635, 2013.

OLIVEIRA, M. I. V.; PEREIRA, E. M.; PORTO, R. M.; LEITE, D. D. F.; FIDELIS, V. R. L.; MAGALHAES, W. B. Avaliação da qualidade pós-colheita de hortaliças tipo fruto, comercializadas em feira livre no município de SolâneaPB, pinBrejo Paraibano. Agropecuária Técnica, Areia-PB, v.37, n.1, p.13-18, 2016a.

OLIVEIRA, D. E. C.; RESENDE, O.; COSTA, L. M. Efeitos da secagem na coloração dos frutos de baru (Dipteryx alata Vogel). Revista Agro@mbiente, Boa Vista-RR, v.10, n.4, p.364-370, 2016 b.

PEREIRA, J. M. A. T. K.; OLIVEIRA, K. A. M.; SOARES, N. F. F.; GONÇALVES, M. P. J. C.; PINTO, C. L. O.; FONTES, E. A. F. Avaliação da qualidade físico-química, microbiológica e microscópica de polpas de frutas congeladas comercializadas na cidade de Viçosa-MG. Alimentos Nutrição, Araraquara-SP, v.17, n.4, p.437-42, 2006.

PINSETTA JUNIOR, J. S. Recobrimento comestível com hidroxipropilmetilcelulose e agentes antiescurecimento em berinjela minimamente processada. Mestre em Agronomia (Produção Vegetal). Universidade Estadual Paulista - Unesp, Câmpus de Jaboticabal. Jaboticabal-SP, 58 p., 2018.

REIS, A. Círculo de hospedeiras de isolados de Stemphylium solani/Ailton Reis, Leonardo Silva Boiteux. - Brasília: Embrapa Hortaliças, Brasília-DF, 13 p., 2006.

RUFINO, M. S. M.; ALVES, R. E.; BRITO, E. S.; MORAIS, S. M.; SAMPAIO, C. G.; PÉRZJIMÉNEZ, J.; SAURACALIXTO, F. D. Metodologia cientifica: Determinação da atividade antioxidante total em frutas pela captura do radical livre DPPH. EMBRAPA: Comunicado Técnico, Fortaleza-CE, 127 p., 2007.

SAATH, K. C. O.; FACHINELLO, A. L. Crescimento da demanda mundial de alimentos e restrições do fator terra no Brasil. Revista de Economia e Sociologia Rural, Brasília-DF, v.56, n.2, 2018.

SHUKLA, V.; L.B. NAIK. Agrotechniques for solanaceous vegetables, In: Advances in Horticulture, v.5. Malhotra Publishing House, New Delhi, India, p.364-399, 1993.

SOUSA, I. M.; GARCIA, L. G. C.; PEIXOTO, J. V. M.; NASCIMENTO, L. M.; SILVA NETO, C. M.; PONTES, N. C. Avaliação do diâmetro e dos parâmetros $a^{*}, b^{*}, L^{*}$ e croma em tomateiro industrial. IV Congresso Estadual de Iniciação Científica do IF Goiano, Goiano-GO, 2 p., 2015.

TENUTA, N. Produtos orgânicos e de base agroecológica: onde encontrar?. 2017. Disponível em: http://mds.gov.br/caisan-mds/educacao-alimentar-e-nutricional/produtos-organicos-e-de-base-agroecologica. Acesso em: 20/04/2019.

VASCONCELOS, T. B.; CARDOSO, A. R. N. R.; JOSINO, J. B.; MACENA, R. H. M.; BASTOS, V. P. D. Radicais livres e antioxidantes: Proteção ou perigo. Científica Ciências Biológicas e da Saúde, Londrina-PR, v.16, n.3, p.213$219,2014$.

WATERHOUSE, A. L. Determination of total phenolics, in Current Protocols. Food Analytical Chemistry, p.111-118, 2002.

\section{AGRADECIMENTOS}

Apoio financeiro: Fundação de Apoio à Pesquisa do Estado da Paraíba - FAPESQ. 